\title{
Monte Carlo Simulation of Magnetization Reversal in Fe Sesquilayers on W(110)
}

\author{
M. Kolesik \\ Supercomputer Computations Research Institute, Florida State University, Tallahassee, Florida 32306-4052 \\ and Institute of Physics, Slovak Academy of Sciences, Dúbravská cesta 9, 84228 Bratislava, Slovak Republic \\ M. A. Novotny \\ Supercomputer Computations Research Institute, Florida State University, Tallahassee, Florida 32306-4052 \\ and Department of Electrical Engineering, 2525 Pottsdamer Street, Florida A $6 M$ University-Florida State University, \\ Tallahassee, Florida 32310-6046 \\ Per Arne Rikvold \\ Center for Materials Research and Technology, Department of Physics, \\ and Supercomputer Computations Research Institute, Florida State University, Tallahassee, Florida 32306 -3016
}

(August 14, 2018)

Iron sesquilayers grown at room temperature on W(110) exhibit a pronounced coercivity maximum near a coverage of 1.5 atomic monolayers. On lattices which faithfully reproduce the morphology of the real films, a kinetic Ising model is utilized to simulate the domain-wall motion. Simulations reveal that the dynamics is dominated by the second-layer islands, which act as pinning centers. The simulated dependencies of the coercivity on the film coverage, as well as on the temperature and the frequency of the applied field, are very similar to those measured in experiments. Unlike previous micromagnetic models, the presented approach provides insight into the dynamics of the domain-wall motion and clearly reveals the role of thermal fluctuations.

PACS Number(s): 75.70.Ak, 75.40.Mg, 64.60.Qb, 05.50.+q.

\section{INTRODUCTION}

Recently, there has been muchinterest in ultrathin iron films on $\mathrm{W}(110)$ substrates. 1 - $\mathrm{B}$ The present work is mainly concerned with the so-called sesquilayers, which are films with coverages between one and two atomic monolayers. When grown at room temperature, such structures consist of a nearly perfect monolayer with compact islands of the second layer on top. The mechanical and magnetif properties are profoundly affected by this morphology. a pronounced maximum as a function of the sesquilayer coverage. In particular, around a coverage of 1.5 monolayers (ML) the coercivity exceeds that of a monolayer or a doublelayer by more than an order of magnitude.t Similarly, elastic properties show an unusual behavior in this thickness range. sence of magnetic long-range order in a certain region of coverage was first interpreted as a manifestation of a spin-glass-like phase 1 However, the required presence of frustrated antiferromagnetic interactions was difficult to justify, and later an pxplanation based on surface roughness was proposed 2 .

The aim of the present article is to study a model of this system by means of computer simulation. Our goal is twofold. First, we provide a semiquantitative explanation for the experimental coercivity observations. In spirit, this is similar to the micromagnetic models proposed in Refs. 2 and 1 , but in contrast to the essentially zero-temperature micromagnetic arguments it gives an insight into the dynamics of the magnetization reversal at nonzero temperatures. Our model reproduces without additional assumptions the experimentally observed temperaturel and frequencyl dependence of the coercivity. Second, we want to demonstrate the usefulness of the Monte Carlo approach to the simulation of real magnetic systems. This is an application-oriented extension of our previous worb on magnetization switching in kinetic Ising models, 10 where one can find the theoretical background for the present article. Some preliminary results of this study have been discussed in Ref. 11 .

The remainder of this paper is organized as follows. In the next section we describe how the dynamic magnetic properties of iron sesquilayers are reflected by our computational model. Section III is devoted to simulation results and their comparison with experimental data. A summary of our results, as well as a brief discussion of the implications of the proposed model for the frequency dependence of the coercivity are given in Sec. IV.

\section{COMPUTATIONAL MODEL OF AN ULTRATHIN IRON FILM}

From the theoretical point of view, an iron monolayer deposited on a W(110) substrate is a nearly ideal ferromagnetic Ising-like system. 1 Although this only implies that the critical fluctuations in the vicinity of the Curie temperature can be described by the Ising model, one is tempted to speculate that it could be used also away from the phase transition. However, what is more important at lower temperatures is the magnetic anisotropy. The 
magnetocrystalline anisotropy of the distorted bcc iron structure on the tungsten substrate is almost a hundred times larger than in bulk iron. The strength of the resulting anisotropy field, on the order of a few $\mathrm{T}, \mathrm{t}$ makes the Ising model a reasonable approximation for this system. The Ising Hamiltonian is given by

$$
\mathcal{H}=-J \sum_{\langle i j\rangle} s_{i} s_{j}-\mu H \sum_{i} s_{i}
$$

Here, the two-state variables $s_{i}= \pm 1$ at the nodes of a computational lattice represent local magnetic moments pointing in the positive or negative in-plane easy-axis [110] directions, respectively. The first sum runs over all nearest-neighbor pairs in the lattice. One and two layers of a square lattice are used to model mono- and double-layer regions of the film, respectively. Therefore, the number of nearest neighbors for a given site depends on its environment. The ferromagnetic spin-spin interaction, $J=8.73 \mathrm{meV}$, is fixed such that the critical temperature, $T_{c}=230 \mathrm{~K}$, of a monolayer $\mathrm{B}$ is correctly reproduced by the exact value for our square computational lattice. The second term, with the summation over all sites, represents the interaction with the component $H$ of the external magnetic field along the easy axis, and $\mu$ is the magnetic moment of the computational spin $s_{i}$. The latter depends on the model lattice spacing $a$, which we choose to be $a=6 \AA$. With this value of $a$, a computational spin $s_{i}$ represents a part of an iron monolayer containing approximately 5 atoms and carries a magnetic moment of $\mu=11.43 \mu_{B}$. We use the bulk value of the iron atom magnetic moment.

The morphology of the iron sesquilayers has been well studied by scanning tunneling microscopy (STM), and high-resolution images of their surfaces are available. Since the roughness of the surface plays a crucial role in the magnetic behavior, we have chosen to digitize STM pictures from Ref. 11 to generate our computational lattices. This has the advantage that the lattices faithfully reproduce the real films. Figure 1 shows the morphology of the iron sesquilayers.

The dynamics we use in our simulations is the standard Glauber Monte Carlo dynamics with local updates at randomly chosen sites, which can be related to the quantum mechanical dynamics.12 To speed up the simulations in weak external fields, we use a variant of the rejection-free Monte Carlo algorithm described in Ref. 13. The simulation time is measured in Monte Carlo Steps per Spin (MCSS). Although its precise relation to physical time is not known, it is expected that one MCSS roughly corresponds to a typical inverse phonon frequency. Here, we fix the simulation time scale such that $1 \mathrm{MCSS} \equiv t_{\mathrm{MC}}=10^{-12} \mathrm{~s}$.

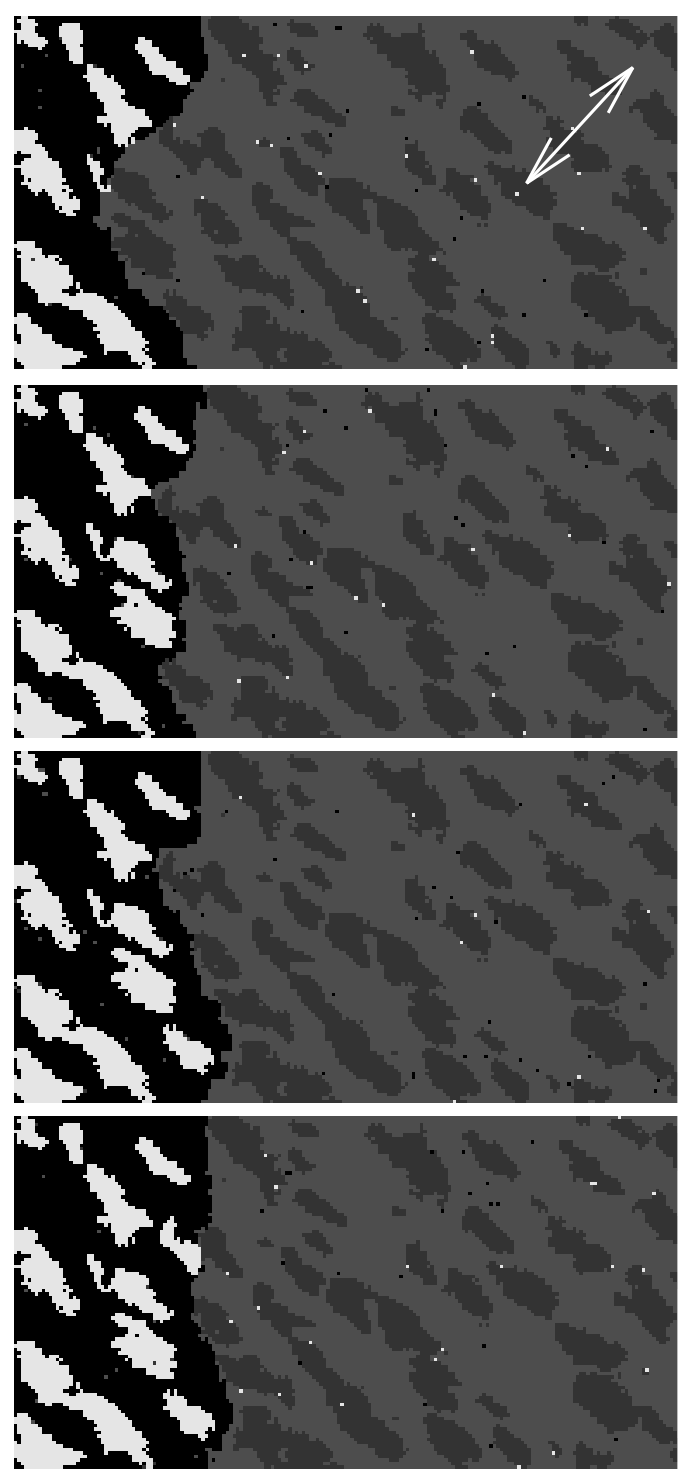

FIG. 1. Illustration of the sesquilayer morphology at a coverage of $1.26 \mathrm{ML}$, with snapshots of the propagating domain wall at the times marked by arrows in Fig. 2. Time increases from top to bottom in the picture. The patches are the islands of the second iron monolayer on top of the first one. The area shown is $1170 \AA \times 610 \AA$, and the island configuration was digitized from Fig.1 j) of Ref. 1. The size of the computational lattice was $195 \times 102 \times 1(2)$ (two layers for islands). $T=1.3 \mathrm{~J} / k_{\mathrm{B}} \approx 132 \mathrm{~K}, \mu H=0.02 \mathrm{~J}$ or $H \approx 0.26 \mathrm{~T}$. The high-contrast region is the stable magnetic phase. The double arrow shows the easy-axis direction. An animation of this simulation can be found at http://www.scri.fsu.edu/ $\sim$ rikvold. 
Before describing how we estimate the coercivity from simulations, let us consider the possible magnetization reversal modes in real systems. There are two extremes for magnetization switching: driven either by nucleation or by domain-wall motion. What is observed in experiments on these ultrathin iron films is the second mechanism. On a microscopic scale, the sample is "infinite," and somewhere there exist seeds of the stable phase which start to grow almost instantaneously when the field is reversed. As a result, magnetization reversal in a typical region of the sample is caused by a domain wall which propagates across the observed area. When the driving magnetic field is weak and/or the disorder is strong, the domain wall is pinned and does not move on the experimental time scale. Thus, the coercivity can be identified with the field which makes the domain wall mobile under given circumstances (disorder, temperature, time scale, ...). To exclude the nucleation time from our simulations, we prepare the initial state with all spins equal to +1 , except for those in a narrow strip along one of the short sides of the lattice. These spins are initialized to -1 and represent a domain of the stable magnetization that has just propagated into the region under observation. We apply a negative magnetic field and measure the time needed, $t_{\mathrm{sw}}$, to reach the state with $90 \%$ of the spins reversed. Since the reversal occurs by propagation of the domain wall, the change in the the reversed volume divided by the lattice width gives us the average distance $D$ the domain wall traveled. Together with the elapsed time it can be used to estimate the average domain-wall velocity.

\section{SIMULATION RESULTS}

Figure 1 illustrates the propagation of a domain wall through a sesquilayer system as it was recorded during a particular simulation run. One notices that the domain wall typically does not cross the second-monolayer patches. Instead, it feels an island as an obstacle and gets pinned near its boundary. To overturn the spins in the island, it is necessary to overcome a free-energy barrier which depends on the driving field, the temperature, and also on the shape and size of the island. Due to thermal fluctuations, the domain wall tries to enter the islands, but is usually driven back by the free-energy barrier. A "successful" fluctuation must be large enough to have greater probability to grow through the island than to shrink and vanish; a fluctuation which just meets this criterion is called critical.

To estimate the size of a critical fluctuation, denote by $\ell$ the linear extent of the region which reverses its magnetization during a jump of the domain wall to its next metastable configuration. The interface energy change $\delta E_{\Sigma}$ is proportional to $\ell$, while the "volume" energy change $\delta E_{H}$ is proportional to $H \ell^{2}$. While the latter is negative, the interface energy change can be negative or positive depending on whether the domain-wall length decreases or increases. The mobility of the domain wall is essentially given by the slower processes, for which $\delta E_{\Sigma}>0$ and $\delta E_{H}<0$. The new configuration will be stable with respect to the previous one only if the volume energy change is sufficient to compensate for the interface energy change. From this it follows that $\ell \sim 1 / H$. Since the interface and volume energies of the intermediate unstable domain-wall configurations scale in the same way, the free-energy barrier $\Delta F$ to be overcome will be inversely proportional to the driving field, $\Delta F=\Xi /(\mu H)$. The field-independent quantity $\Xi$ depends on the local environment, as well as on the temperature. In such a situation, nucleation theory predicts that the waiting time needed to observe a critical fluctuation is 10

$$
\tau \sim H^{-K} \exp \left(\frac{\Xi}{k_{\mathrm{B}} T \mu H}\right),
$$

where the exponent $K$ is taken to be equal fo 3 as expected for the two-dimensional Ising model, $\theta$ and $k_{\mathrm{B}}$ is Boltzmann's constant. Once a critical fluctuation is created, the domain wall reorganizes quickly until it reaches a new metastable configuration of lower free energy than the previous one. In this way, propagation of a domain wall through the sesquilayer is explained as a series of thermally induced nucleation events followed by rapid magnetization changes.

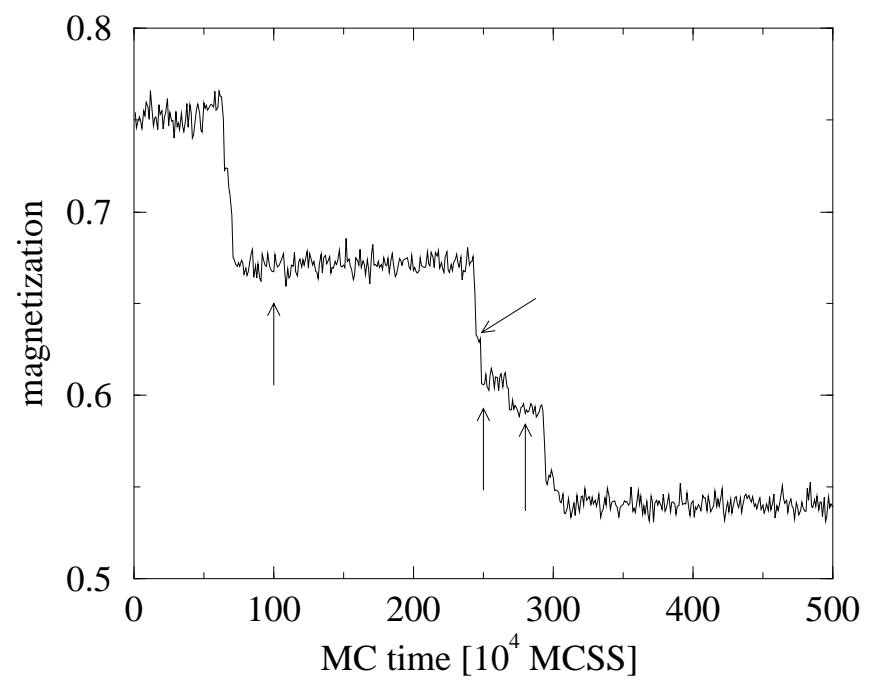

FIG. 2. Magnetization per spin vs. time during the same simulation from which the snapshots in Fig. 1 were taken (at the times indicated by arrows). Most of the time, the magnetization remains constant, apart from small fluctuations. The movement of the domain wall occurs in jumps. Each jump represents a nucleation event, after which the domain wall advances quickly to its new metastable configuration.

Figure 2 shows how the intermittent domain-wall motion is reflected in the magnetization per spin. The horizontal parts of the plot represent situations in which 
the domain wall remains unchanged, apart from small fluctuations. The steps correspond to events when critical fluctuations appear, thereby causing small regions of the sample to reverse their magnetization into the stable phase. The average time between the magnetization jumps naturally depends on the size of the sample observed. For large regions it is expected to scale as the inverse of the length of the domain wall. Similarly, the size of an individual magnetization jump is proportional to the fraction of the observation area that is switched during that jump. Therefore, the magnetization curve should appear smooth for observation areas much larger than a typical island size.

It is interesting to note that similar jumps in the magnetization have been observed experimentally in some thin magnetic films.14 Although in the present case the typical size of these Barkhausen jumps is about ten times smaller than in that study, it is possible that the effect could be observed using similar magneto-optical techniques.

While traversing the sample, the domain wall encounters barriers of different heights, and the total time needed to travel a certain distance is the sum of contributions similar to Eq. (2), but with different values of $\Xi$. As our simulations show, the residence time in different metastable domain-wall configurations can be very different, suggesting that there is a broad distribution of free-energy barriers. Since we cannot determine that distribution, we assume it to be uniform between zero and an upper cutoff $\Delta$. This and Eq. (2) lead us to an approximate formula for the time elapsed during the domain-wall propagation through a given sample (we only keep the dominant terms),

$$
t_{\mathrm{sw}} \approx \frac{A}{\mu H}+\frac{B}{(\mu H)^{2}} \exp \left(\frac{\Delta}{k_{\mathrm{B}} T \mu H}\right) .
$$

Here we have added the first term, which corresponds to the time elapsed during the "free" domain-wall propagation, when the velocity is proportional to the driving magnetic field $H$. This term is usually negligible, except in systems with coverage close to two. The parameters $A, B$ and $\Delta$ are fit to the simulation data as described below.

The effective domain-wall velocity $v_{\text {eff }}=D / t_{\mathrm{sw}}$ (with $D$ standing for the traveled distance, defined as the switched volume divided by the width of the lattice), obtained from the averaged switching times, is shown in Fig. 3 as a function of the driving field for several different coverages of the sesquilayer systems. A qualitatively similar domain-wall velocity dependence on driving field was found in experiments on magnetic films, $15-47$ in which the domain-wall motion is also believed to be controlled by thermal fluctuations.

What is shown in Fig. 3 is an analogue of a depinning transition rounded by a finite temperature. It is to be stressed that in the present case there is no sharp transition, even in an arbitrarily weak field. Strictly speaking, the average domain-wall velocity is always nonzero, but it quickly approaches zero on a scale of field strengths which, in agreement with the experimental findings, depend nonmonotonically on the coverage.
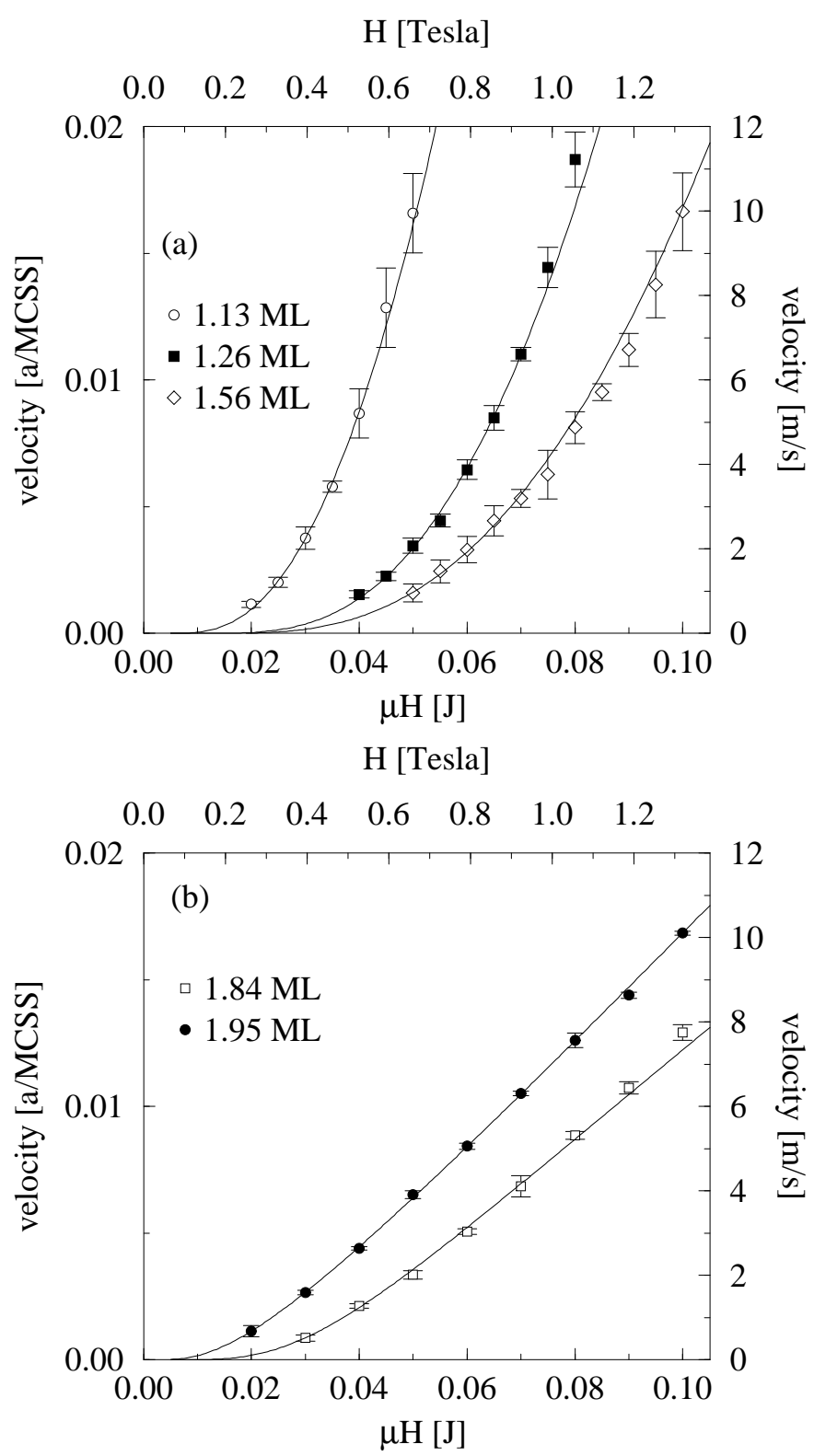

FIG. 3. Average domain-wall velocity as a function of the driving magnetic field for several different coverages at $T=184 \mathrm{~K}$. As the coverage increases towards $\approx 1.56 \mathrm{ML}$, the field near which the velocity practically vanishes increases (a). For coverages closer to $2 \mathrm{ML}$, it decreases again with increasing coverage (b).

We measured the switching times for several sesquilayer samples with various coverages as functions of the magnetic field. Using Eq. (3), we estimated the fitting parameters for each coverage. The resulting val- 
ues are summarized in Table I. We note that the behavior of the fitting parameters as functions of the coverage is quite reasonable. Also, the effective velocity of the "free domain-wall motion" $D \mu H / A$ is similar to what has been measured in pure Ising systems 18

We want to point out the difference between our Eqs. (2, 3) and an approach commonly used in the experimental literature 15,16 in which one supposes that the free-energy barriers decrease linearly with $H$. That would be appropriate only if the size of the critical fluctuations were fixed. The scaling argument leading to Eq. (2) implies that that is not so in the present case. However, it is extremely difficult to distinguish numerically between linear and inverse linear dependencies if one only has data from a relatively narrow range of field strengths. That is usually the case in experiments, and we face the same problem here. However, attempting to fit our simulation results using a linear field dependence, we obtain fitting parameters which behave rather erratically as functions of the coverage. We therefore believe that the " $1 / H$ model" used here is more appropriate in the range of $H$ studied here, also from the numerical point of view.

Another question concerning the functional form of the velocity-field relation used here, is whether it may be extrapolated to fields sufficiently weak that the velocity vanishes on the experimental time scale. We know that in a relatively strong field the size of the critical fluctuation $l$ varies as $l \sim 1 / H$. But is this still true when $l$ exceeds the length scales characteristic of the island structure of the film? Or, equivalently, is the disorder strong enough to provide for a kind of "weak pinning" on large length scales, with groups of islands acting as "collective" pinning centers? Both qualitative arguments and exploratory simulations of simplified configurations indicate that this is the case. However, the coercivities extracted from our simulations approximately correspond to magnetic fields in which $l$ approaches the island size. We cannot exclude that in even weaker fields the relation of the velocity to $H$ is characterized by parameters different from those obtained in the relatively strong-field region to which our simulations are confined. Without being able to perform simulations in extremely weak fields, the only way to judge the appropriateness of our extrapolation is the comparison of our final results with the experimental data. The good, semiquantitative agreement is an indication that our expectation is, indeed, correct.

Having an approximation formula for the domain-wall velocity $v_{\text {eff }}(H)$ as a function of the field, we can estimate the coercivity $H_{c}$. Since coercivity is usually measured in a time-dependent field, we have to translate our static measurements into such a situation. We restrict ourselves to relatively slowly varying and weak fields in which the domain-wall motion is the main magnetization switching mechanism. Suppose that the system is subjected to a magnetic field $H_{0} \sin (\omega t)$. The average domain-wall displacement at time $t$ is then $\int_{0}^{t} v_{\text {eff }}\left(H_{0} \sin \left(\omega t^{\prime}\right)\right) d t^{\prime}$. Denote by $L_{\mathrm{S}}$ the typical distance between seeds of the sta- ble phase. Then, the coercivity can be estimated as the value of the driving field reached when the domain walls have traveled that distance,

$$
\frac{a}{t_{\mathrm{MC}} L_{\mathrm{S}} \omega} \int_{0}^{\arcsin \left(H_{c} / H_{0}\right)} v_{\mathrm{eff}}\left(H_{0} \sin (\phi)\right) d \phi \approx 1 .
$$

The velocity $v_{\text {eff }}$ is given in dimensionless units, and the conversion factor to $\mathrm{ms}^{-1}, a / t_{\mathrm{MC}}$, is shown explicitly. Note that this equation is determined up to a factor of order unity. Since $v_{\text {eff }}$ depends strongly on $H$, this does not affect the estimated coercivity significantly. It is the order of magnitude of the combination $a /\left(t_{\mathrm{MC}} L_{\mathrm{S}} \omega\right)$ and the value of the barrier $\Delta$ which determine the coercivity.

There are two quantities in our model which we cannot determine precisely, namely the typical distance $L_{\mathrm{S}}$ between the stable-domain seeds, and the factor $t_{\mathrm{MC}}$ which relates the Monte Carlo and the physical time scales. They always enter our formulas as a product, so there is a single undetermined parameter in the theory which can be regarded as a fitting parameter fixing the coercivity scale. We use the following values for $t_{\mathrm{MC}}, L_{\mathrm{S}}$, and $\omega: t_{\mathrm{MC}}=10^{-12} \mathrm{~s}, L_{\mathrm{S}}=10^{-5} \mathrm{~m}$, and $\omega=2 \pi \mathrm{s}^{-1}$. While these are "reasonable" values, they were simply chosen such that the coercivity simulated at $0.8 T_{c}(184 \mathrm{~K})$ exhibits a maximum value (as a function of coverage) which roughly corresponds to the experimental observation.

We used $H_{0}=0.5 \mathrm{~T}$ for the value of the driving-field amplitude, which is about the field strengths used in experiments. 3 . 3 When the coercivity $H_{c}$ is much smaller than $H_{0}$, it depends only weakly on the amplitude and frequency of the driving field in the combination $\omega H_{0}$. On the other hand, one observes much stronger dependence on the amplitude when $H_{c}$ and $H_{0}$ are comparable. In the extreme case when $H_{0}$ is less than the coercivity, there is no solution to our Eq. (4). This situation corresponds to the experimentally observed collapse of the hysteresis loop $\mathrm{g}$

In Fig. 4 we plot the coercivity $H_{c}$ as a function of the sesquilayer coverage. The coercivity first steeply increases to a maximum at a coverage of about 1.4. Then it decreases as the coverage approaches a full double layer. This is very similar to the experimentally observed behavior of the coercivity. As the temperature decreases, the coercivity rapidly increases. Sander et al. reported a coercivity higher than $0.3 \mathrm{~T}$ at $140 \mathrm{~K}$ O Our simulation in that temperature range nicely reproduces that observation. To illustrate the effect of the driving-field frequency $\omega$, we also show a coercivity curve (filled circles) predicted for a measurement at a frequency 1000 times higher than the one shown by open circles.

It is expected that another coercivity maximum would be observed for coverages between 2 and 3, although it should be much less pronounced. We do not have suitable experimental data to create lattice structures for this coverage region, but simulations on structures which simply repeat the morphology of the thinner films do suggest the existence of the second maximum. 


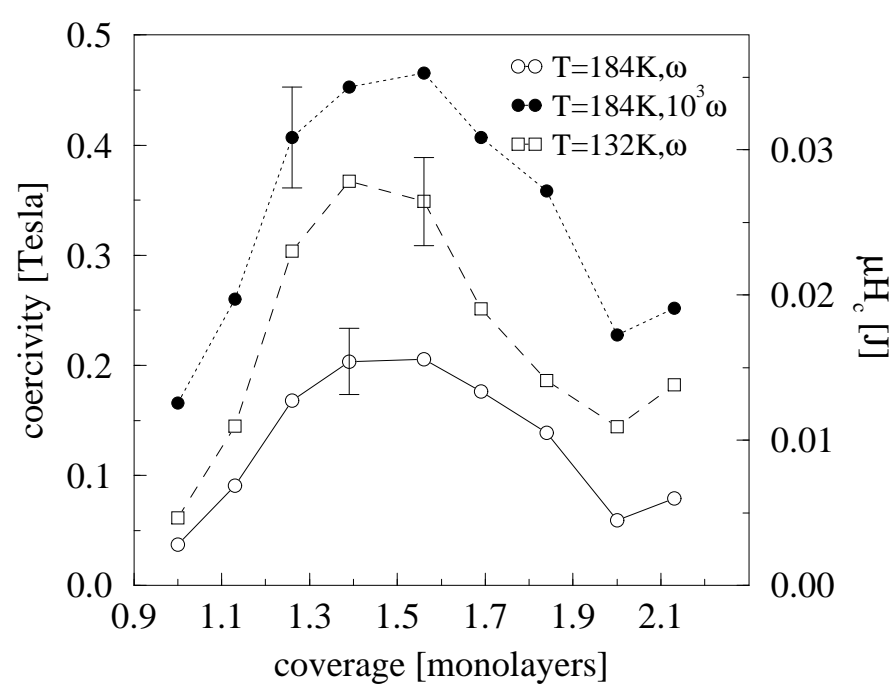

FIG. 4. Coercivity, $H_{c}$, of the iron sesquilayer as a function of coverage. Different curves represent measurements at different temperatures and frequencies. The lower curve is to be compared with Fig. 3 of Ref. 1 . Representative error bars are shown to give a rough idea of the statistical uncertainty due to errors in the fitting parameters.

\section{CONCLUSION}

In conclusion, the good agreement between the shapes and the dependencies on $T$ and $\omega$ of the simulated and experimental plots of coercivity vs. coverage suggests that a simple kinetic Ising model can describe the basic processes involved in domain-wall motion at nonzero temperatures in highly anisotropic films. Our simulations corroborate that the coercivity maximum observed for iron sesquilayers on $\mathrm{W}(110)$ is caused by domain-wall pinning at the islands of the second monolayer. Although the pinning mechanism here is in principle similar to the one proposed in the micromagnetic model of Ref. 2, the crucial difference is in the role of thermal fluctuations, which the present model takes into account. Thermal fluctuations allow the domain wall to overcome obstacles created by the second-monolayer islands by creating critical fluctuations of the domain-wall shape. The domain-wall motion is intermittent with long periods of quiescence punctuated by nucleation events which are followed by rapid forward jumps of the wall. Without any further assumptions, our model also reproduces an increase in the coercivity at lower temperatures. It is to be stressed that there is only one unknown parameter in the model. Once it is fixed, simulations at different temperatures and film coverages can be used to predict the coercivity.

Another interesting point is that the model also predicts how the coercivity depends on the sweep rate (or frequency) of the magnetic field in which the measurement is done. By integrating Eq. (何) in the low-frequency limit one can show that the inverse coercivity depends ap- proximately linearly on the logarithm of the sweep rate (frequency). Figure 5(a) shows that such an approximation is quite reasonable. However, the same data plotted on a $\log \left(H_{c}\right)$ vs. $\log (\omega)$ scale in Fig. $5(\mathrm{~b})$ also provide a quite good linear plot. Recent experiments by Suen et al. 6 show that the logarithm of the hysteresis loop area (which, because of the shape of the loop, can be replaced by the coercivity in this case) increases linearly with $\log (\omega)$ over several decades. Thus, our observation is in good agreement with experimental findings, but it also demonstrates that it can be difficult to draw conclusions about the functional form of the frequency dependence, even with data spanning several frequency decades.
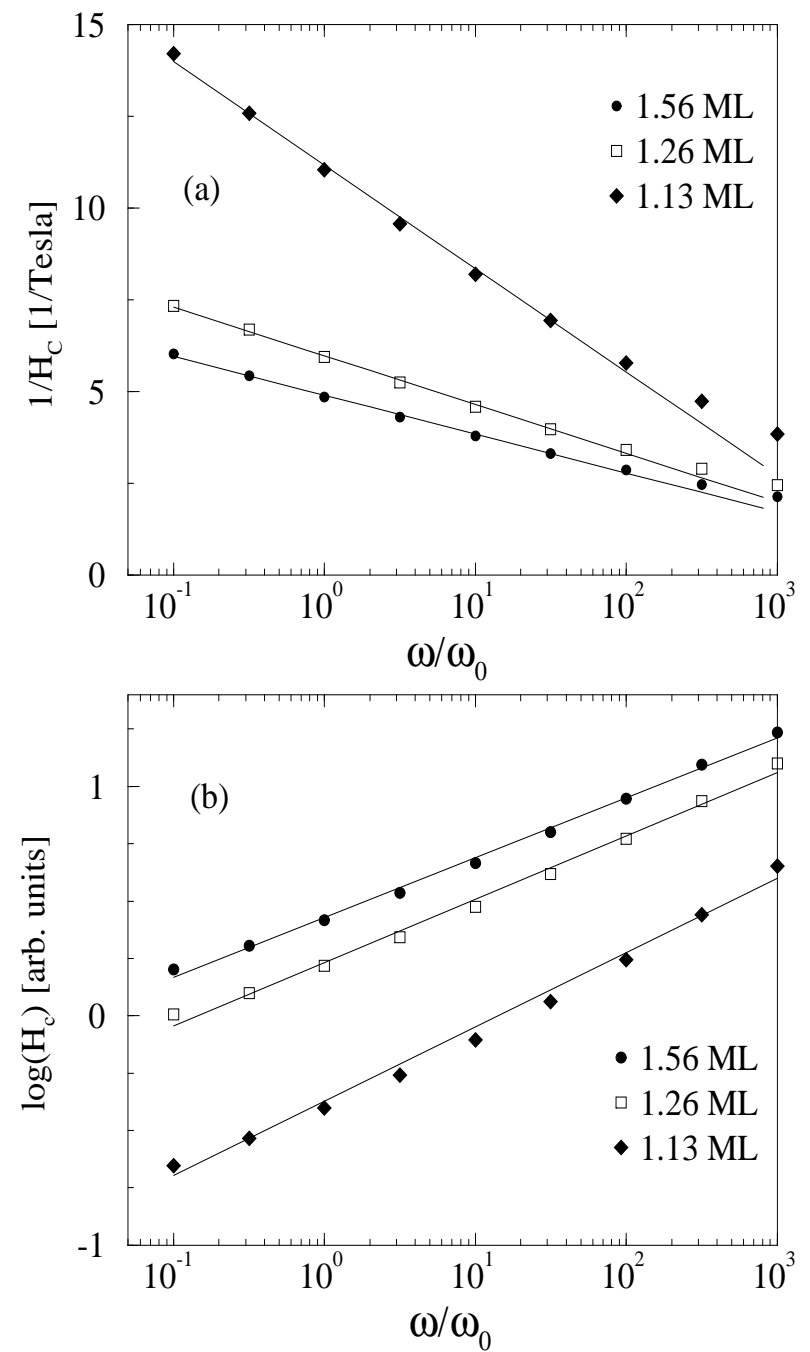

FIG. 5. The proposed model predicts that the coercivity depends on the time scale of the measurement. Approximately, the inverse of the coercivity is linear in the logarithm of the magnetic field sweep rate (frequency), as shown in panel (a). The same data is shown also as $\log \left(H_{c}\right)$ vs. $\log (\omega)$ in panel (b). Note that the plots are nearly linear in both cases. Points represent calculations based on our simulation data, and straight lines are merely guides to the eye. With $\omega_{0}=2 \pi$, the shown frequency range roughly corresponds to the one in Ref. 8 . 
Finally, we note that the proposed model is interesting not only from the point of view of its application to sesquilayer iron films. It describes the basic physics of thermally activated domain-wall motion, which is common to many experimental systems (for example the perpendicularly magnetized ultrathin films studied in Refs. [ 14 17, 19, 20 ) for which, when suitably modified, the kinetic Ising model could provide a tool for a better understanding of many effects. Ising-like models with a higher number of states can be used to study rough films with a four-fold anisotropy 21 or can serve as an alternative to the spin-1 mean-field-type description of the influence of the island structure on the coercivity.22 The dynamics of the model is also closely related to more general topics such as interface propagation in disordered media 23 and depinning transitions in the presence of thermal fluctuations or hysteretic creep of elastic manifolds.24

The authors wish to thank A. Zangwill for useful discussions. This research was supported by NSF Grants No. DMR-9315969 and DMR-9520325, FSUMARTECH, FSU-SCRI (DOE Contract No. DE-FC0585ER25000).

${ }^{1}$ H. Bethge, D. Heuer, Ch. Jensen, K. Reshöft and U. Köhler, Surf. Sci. 331-333, 878 (1995).

${ }^{2}$ D. Sander, R. Skomski, C. Schmidthals, A. Enders and J. Kirschner, Phys. Rev. Lett. 77, 2566 (1996).

${ }^{3}$ D. Sander, A. Enders, R. Skomski and J. Kirschner, IEEE Trans. Magn. 32, 4570 (1996).

${ }^{4}$ R. Skomski, D. Sander, A. Enders and J. Kirschner, IEEE Trans. Magn. 32, 4567 (1996).

${ }^{5}$ H. J. Elmers, J. Hauschild, H. Fritzsche, G. Liu and U. Gradmann, Phys. Rev. Lett. 75, 2031 (1995).

${ }^{6}$ H. J. Elmers, J. Hauschild, H. Höche, U. Gradmann, H. Bethge, D. Heuer and U. Köhler, Phys. Rev. Lett. 73, 898 (1994).

${ }^{7}$ H. J. Elmers and U. Gradmann, Appl. Phys. A 51, 255 (1990).

8 J. H. Suen and J. L. Erskine, Phys. Rev. Lett. 78, 3567 (1997).

${ }^{9}$ P. A. Rikvold, H. Tomita, S. Miyashita and S. W. Sides, Phys. Rev. E 49, 5080 (1994); P. A. Rikvold and B. M. Gorman, in Ann. Rev. Comp. Phys. I, edited by D. Stauffer (World Scientific, Singapore, 1994), p. 149.

${ }^{10}$ H. L. Richards et al., J. Magn. Magn. Mater. 150, 37 (1995); J. Appl. Phys. 79, 5479 (1996); Phys. Rev. B 54, 4113 (1996); Phys. Rev. B 55, 11521 (1997).

${ }^{11}$ P. A. Rikvold, M. A. Novotny and M. Kolesik, preprint cond-mat/9705189.

12 Ph. A. Martin, J. Stat. Phys. 16, 149 (1977).

13 M. A. Novotny, Phys. Rev. Lett. 74, 1 (1995); Erratum 75, 1424 (1995); Comp. in Phys. 9, 46 (1995).

14 S. Gadetsky and M. Mansuripur, J. Appl. Phys. 79, 5667 (1996).
${ }^{15}$ G. V. Sayko, A. K. Zvezdin, T. G. Pokhil, B. S. Vvedensky and E. N. Nikolaev, IEEE Trans. Magn. 28, 2931 (1992).

16 T. G. Pokhil and E. N. Nikolaev, IEEE Trans. Magn. 29, 2536 (1993).

${ }^{17}$ A. Kirilyuk, J. Ferré and D. Renard, IEEE Trans. Magn. 29, 2518 (1993).

${ }^{18}$ R. Ramos, S. W. Sides, P. A. Rikvold, M. A. Novotny, in preparation.

19 Y.-L. He and G.-C. Wang, Phys. Rev. Lett. 70, 2336 (1993).

20 Z. Q. Qiu, J. Pearson and S. D. Bader, Phys. Rev. B 49, 8797 (1993).

21 A. Moschel, R. A. Hyman, A. Zangwill and M. D. Stiles, Phys. Rev. Lett. 77, 3653 (1996).

${ }^{22}$ C. N. Luse and A. Zangwill, J. Appl. Phys. 79, 4942 (1996).

${ }^{23}$ U. Nowak, J. Heimel, T. Kleinfeld and D. Weller, Phys. Rev. B in press.

${ }^{24}$ S. Scheidl and V. Vinokur, Phys. Rev. Lett. 77, 4768 (1996).

\begin{tabular}{|c|c|c|c|}
\hline \hline coverage & $A / D[J]$ & $B / D\left[J^{2}\right]$ & $\Delta / k_{\mathrm{B}} T[J]$ \\
\hline 0.95 & - & $0.08(1)$ & $0.007(7)$ \\
\hline 1.13 & - & $0.08(1)$ & $0.033(2)$ \\
\hline 1.26 & - & $0.16(1)$ & $0.075(5)$ \\
\hline 1.39 & - & $0.15(3)$ & $0.100(15)$ \\
\hline 1.56 & - & $0.22(2)$ & $0.096(3)$ \\
\hline 1.69 & $5.1(5)$ & $0.10(1)$ & $0.090(8)$ \\
\hline 1.84 & $6.2(3)$ & $0.12(2)$ & $0.059(5)$ \\
\hline 1.95 & $4.5(4)$ & $0.12(2)$ & $0.015(4)$ \\
\hline 2.13 & $5.1(5)$ & $0.09(2)$ & $0.026(5)$ \\
\hline \hline
\end{tabular}

TABLE I. Fitting parameters of $v_{\mathrm{eff}}=D / t_{\mathrm{sw}}$ for several coverages at $T=184 \mathrm{~K}$. Because of insufficient data acuracy, only a two-parameter fit was used for low coverages, where the term proportional to $A$ is small compared to the exponential contribution in Eq. (3). Numerical uncertainities were estimated by processing two or more independent data sets. 